Revista Brasileira de Meteorologia, v.23, n.1, 30-34, 2008

\title{
CARACTERIZAÇÃO DA VELOCIDADE E DIREÇÃO PREDOMINANTE DOS VENTOS PARAA LOCALIDADE DE ITUVERAVA-SP
}

\author{
FABRÍCIO CÉSAR MUNHOZ e ANICE GARCIA
}

\author{
Faculdade de Agronomia Dr. Francisco Maeda - FAFRAM \\ Rodovia Jerônimo Nunes Macedo, Km 01 Ituverava - SP \\ Email: anicegarcia@yahoo.com.br
}

Recebido Maio 2006 - Aceito Maio 2007

\begin{abstract}
RESUMO
O objetivo do trabalho foi determinar a direção e velocidade predominante do vento para a região de Ituverava, SP. As direções diárias do vento foram coletadas por meio de Anemógrafo tipo Universal, instalado a $2 \mathrm{~m}$ acima da superfície do solo, localizado na Estação Meteorológica da Faculdade "Dr. Francisco Maeda". A direção predominante do vento foi caracterizada por meio de uma análise de freqüência das observações diárias, para cada um dos doze meses do ano. Na maior parte do ano há predominância de ventos vindos de sudeste. De agosto a novembro os ventos sopram com intensidade superior a média, sendo o mês de setembro aquele com velocidades mais elevadas e o mês de abril com as menores velocidades médias. A velocidade média anual, durante o período diurno foi de 2,20 $\mathrm{m} / \mathrm{s}$ e para o período noturno foi de $1,25 \mathrm{~m} / \mathrm{s}$, indicando um aumento médio de $40 \%$ nas velocidades dos ventos diurnos em relação aos noturnos.

Palavras-chave: velocidade do vento, direção do vento, anemógrafo
\end{abstract}

\begin{abstract}
CHARACTERIZATION OF THE PREDOMINANT WIND SPEED AND WIND DIRECTION AT ITUVERAVA, SÃO PAULO STATE, BRAZIL

The objective of the work was to determine the predominant wind direction and speed for the region of Ituverava, SP, Brazil. The daily directions of the wind were collected through universal anemography, located in the Weather station of University "Dr. Francisco Maeda". In most of the year there is predominance of southeast winds (SE). Between August and November the winds blow with higher intensity than the average, being the month of September that with higher speeds and the month of April with the smallest medium speeds. The annual medium speed for the period of the day was of $2.20 \mathrm{~m} / \mathrm{s}$ and for the night period it was of $1.25 \mathrm{~m} / \mathrm{s}$ indicating a medium increase of $40 \%$ in the speeds of the winds of the day in relation to the nocturnes.
\end{abstract}

Keywords: Wind speed, Wind direction, Anemograph

\section{INTRODUÇÃO}

A diferença de gradiente de pressão atmosférica gera o deslocamento do ar, ou seja, origina os ventos. Estes, partindo de zonas de maior para as de menor pressão, sofrem influências também do movimento de rotação da Terra, da força centrífuga ao seu movimento, bem como da topografia e conseqüente atrito com a superfície terrestre (TUBELIS \& NASCIMENTO, 1984).

O vento é uma das variáveis meteorológicas mais importantes e menos estudada, sendo que no caso da agricultura, é conhecida a sua influência na aplicação de defensivos e em estudos voltados à propagação de doenças, polinização e práticas com quebra-vento. Ainda no meio agrícola é crescente a necessidade de informações para subsidiar projetos que buscam o aproveitamento do vento para a construção de secador solar e ligados à economia de água. Outro aspecto positivo é a utilização do vento como fonte de energia renovável (energia eólica). Em relação às plantas, sabe-se que o vento afeta o crescimento destas de três maneiras: transpiração, absorção de $\mathrm{CO}_{2}$ e efeito mecânico sobre as folhas e ramos, sendo que o resultado desta interação depende da espécie.

Além da intensidade do vento, deve-se dar atenção também à sua direção predominante. A localização de proteções 
vegetais (quebra-vento), tão úteis nas atividades agropecuárias, depende principalmente do conhecimento das direções predominantes do vento. Na zona urbana, o conhecimento destas informações permite a correta instalação de indústrias, de modo que não prejudiquem as áreas residenciais.

A direção do vento é bastante variável no tempo e no espaço, em função da situação geográfica do local, da rugosidade da superfície, do relevo, da vegetação e da época do ano (VENDRAMINI, 1986).

Segundo MARTINS (1993), o conhecimento da direção predominante dos ventos e velocidades médias que ocorrem num local fornece informações importantes para o posicionamento de quebra ventos, orientações na construção de estábulos, distribuição das diferentes culturas no campo e principalmente, no posicionamento e dimensionamento das torres para a utilização desta fonte de energia natural.

SILVA et al. (1997) realizaram estudos sobre direção e velocidade dos ventos em Pelotas-RS e concluíram que a direção predominante dos ventos varia com a estação do ano; na primavera e no verão a direção predominante é leste $(E)$, no outono a direção é sudeste (SE) e de nordeste (NE) no inverno. Segundo estes autores em todas as estações do ano, há uma grande incidência de ventos de direção nordeste (NE).

Trabalhos de MACHADO (1950) e REIS \& BERLATO (1972) mostraram que, para todas as regiões climáticas do Estado do Rio Grande do Sul, a direção predominante dos ventos é do quadrante leste: NE, E e SE. As velocidades médias mais elevadas ocorrem nos meses de primavera e as menores no outono.

GALVANI et al. (1999) observaram que a direção predominante de ventos diurnos em Maringá foi Nordeste, e para noturnos, Sudeste (de janeiro a maio e de outubro a dezembro) e a Leste (de julho a setembro). MORENO (1961) constatou que a direção normal predominante para Santa Maria - RS é leste (E). Leste e sudeste também são as direções predominantes dos ventos em Piracicaba (Wiendle e Angelocci (1995) citados por PEREIRA et al., 2002).

Considerando-se a importância de se conhecer o comportamento do vento, realizou-se este trabalho, cujo objetivo foi o de caracterizar a velocidade e direção predominante dos ventos para a região de Ituverava - SP

\section{MATERIAL E MÉTODOS}

Foram utilizados dados diários da direção e velocidade dos ventos coletados no período de 1998 a 2002 no posto meteorológico da Fundação Educacional de Ituverava Faculdade “Dr. Francisco Maeda", em Ituverava, SP. As coordenadas geográficas são: latitude $20^{\circ} 20^{\prime} 31^{\prime \prime} \mathrm{S}$, longitude $47^{\circ} 46^{\prime} 07^{\prime \prime}$ W e altitude de $619 \mathrm{~m}$, com temperatura média anual de $22,8^{\circ} \mathrm{C}$ e precipitação média anual de $1569,3 \mathrm{~mm}$. O clima da região, de acordo com a classificação de Köppen, é Aw, com verão úmido e inverno seco (CARRER e GARCIA, 2007). Utilizaram-se observações efetuadas por meio de anemógrafo universal, instalado a $2 \mathrm{~m}$ acima da superfície do solo. Os valores de velocidade e direção dos ventos foram cotados dos anemogramas de hora em hora considerando-se os períodos de 0 à $1 \mathrm{~h}, 1$ às $2 \mathrm{~h}, 2$ às $3 \mathrm{~h}$ e assim sucessivamente até às $24 \mathrm{~h}$.

A direção predominante do vento foi caracterizada por meio de uma análise de freqüência das observações diárias, para cada um dos doze meses do ano, utilizando-se a seguinte expressão:

$$
f(x)=\frac{n}{N} .100
$$

em que, $\mathbf{f}(\mathbf{x})$ - freqüência de ocorrência do vento em uma determinada direção; $\mathrm{n}$ - numero de ocorrências de uma determinada direção; $\mathrm{N}$ - número total de observações.

As freqüências de direção foram plotadas em gráficos do tipo rosa dos ventos para uma melhor visualização.

As conversões das velocidades médias diárias para altura de 10 metros foram feitas através da equação proposta por PASQUILL (1949) citado por WAGNER (1989):

$$
\left(\frac{U_{2}}{U_{1}}\right)=\left(\frac{z_{2}}{z_{1}}\right)^{0,143}
$$

Em que U2 e $\mathrm{U}_{1}$ são as velocidades para as alturas z2 $(10 \mathrm{~m}) \mathrm{e}$ z1 $(2 \mathrm{~m})$, respectivamente, sendo portanto possível simplificar a equação, obtendo-se:

$$
U_{10}=U_{2} \cdot 1,26
$$

\section{RESULTADOS E DISCUSSÃO}

\subsection{Direção}

A Figura 1 apresenta a direção média do vento em porcentagem para os doze meses do ano, durante o período de 1998 a 2002. Analisando-se esta figura, percebe-se que durante o ano, o vento de sudeste (SE) predominou a maior parte do ano, com exceção do período de verão (dezembro, janeiro e fevereiro), em que essa predominância foi dividida com ventos de noroeste (NW). 


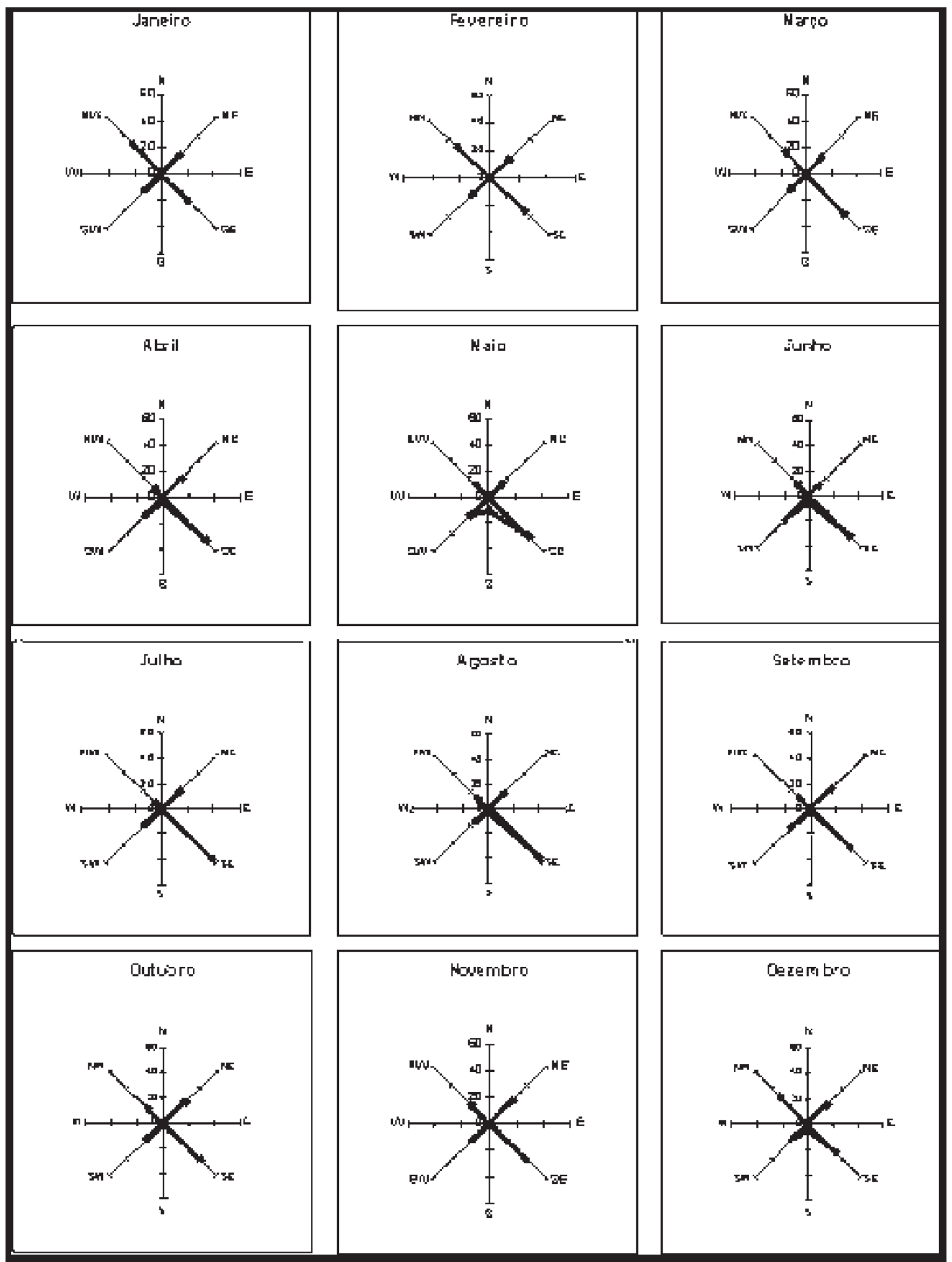

Figura 1. Freqüência média da direção do vento para a Região de Ituverava, SP, nos doze meses do ano, no período de 1998 a 2002. 
Pode-se verificar que, com exceção do mês de janeiro, no qual a maior freqüência $(31 \%)$ das observações foi de vento $\mathrm{NW}$, em todos os outros meses predominou vento SE, com valores de freqüência variando ente $30 \%$ em dezembro (mês no qual a freqüência da direção NE também atingiu 30\%) a 56\% em julho. Tais ventos, de noroeste e de sudeste no hemisfério do sul são genericamente conhecidos como ventos Alísios e estão relacionados ao Centro de Alta Pressão do Atlântico. A posição e a intensidade do centro de alta pressão atmosférica, modifica-se sazonalmente, alterando consequentemente também o padrão de ventos. Este sistema de ventos tem duas direções principais: nordeste e leste durante a primavera e o verão e sudeste durante o outono e o inverno. Isso ocorre devido a migração, para norte, da célula anticiclone do Atlântico Sul no verão, e para sul no inverno (NIMER 1989). Durante o inverno o avanço para norte de frentes frias polares dá força aos ventos de sudeste, adicionando uma componente sul-sudoeste na circulação atmosférica.

A freqüência de ventos de noroeste (NW) apresentam-se num padrão menor durante o outono e inverno e aumentando relativamente durante a primavera, e atingem a maior freqüência durante o verão.

Assim, as freqüências de nordeste (NE) e sudoeste (SW) são as que se apresentam em segundo lugar na maioria dos meses, exceto nos meses de dezembro a março, nos quais o segundo lugar em freqüência é o de direção noroeste (NW).

\subsection{Velocidade média}

A Figura 2 mostra os valores de velocidade média para os 5 anos estudados.
Com relação à diferença entre ventos diurnos e noturnos, as velocidades de vento tenderam aumentar de valor no decorrer do dia, desde o nascer do sol, até por volta do meio da tarde, diminuindo novamente após, semelhante ao observado por PARKER et al. (2004) e DAS et al. (1998).

Observa-se que nos meses mais quentes (Set - Fev) os ventos diurnos suplantaram os noturnos entre $36 \%$ a $55 \%$; nos meses mais frios (mar - ago) essa diferença se reduz, mas os ventos diurnos ainda suplantaram os noturnos de $31 \%$ a $53 \%$.

Esta tendência de ventos diurnos maiores que os noturnos deve-se ao aquecimento do solo durante o dia, o qual também vai aquecendo o ar, que ascende, sendo ocupado por um ar mais frio, originando um gradiente térmico que, por sua vez, origina um gradiente de pressão causando o deslocamento do ar da zona de maior pressão para a zona de menor pressão. À noite, como esse gradiente térmico é menor, a velocidade do vento também é menor (SENAMBI, 2004).

$\mathrm{Na}$ análise por estação do ano, destaca-se a primavera com as maiores velocidades $(2,6 \mathrm{~m} / \mathrm{s}$-diurnos e $1,4 \mathrm{~m} / \mathrm{s}$ noturnos), e o outono com as menores velocidades (1,91 $\mathrm{m} / \mathrm{s}$-diurnos e $1,06 \mathrm{~m} / \mathrm{s}$-noturnos), dados semelhantes aos observados por SILVA et al (1997) para Pelotas- RS.

A velocidade média anual foi de $2,20 \mathrm{~m} / \mathrm{s}$ (período diurno) e de $1,25 \mathrm{~m} / \mathrm{s}$ (período noturno), indicando um aumento médio de $40 \%$ nas velocidades dos ventos diurnos em relação aos noturnos. A velocidade média mínima ocorrida no período analisado foi de 0,0 (vento calmo) e as máximas de $2,76 \mathrm{~m} / \mathrm{s}$ (diurno) e $1,76 \mathrm{~m} / \mathrm{s}$ (noturno), ambas ocorrendo no mês de setembro, o que demonstra um comportamento da velocidade dos ventos muito variável.

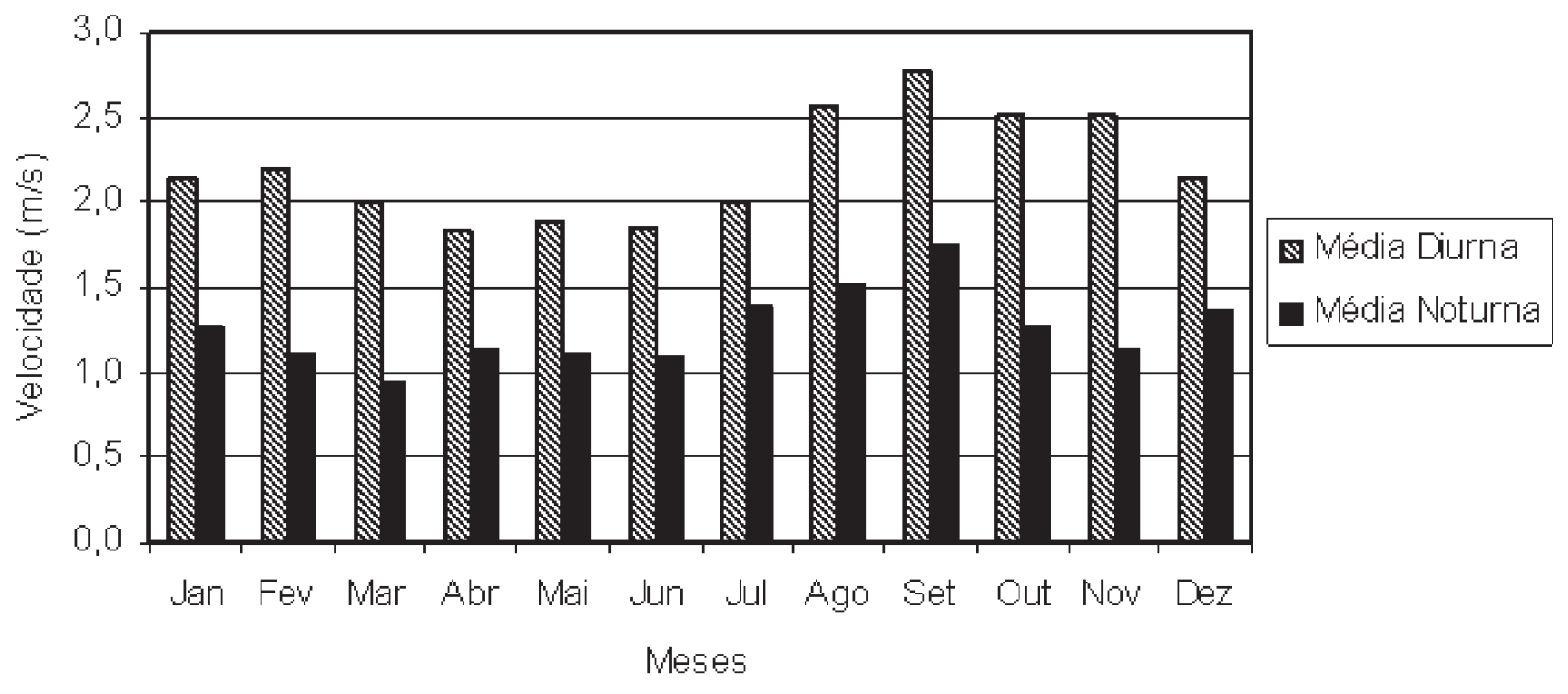

Figura 2. Velocidade média do vento diurno e noturno (em m/s) convertidas a $10 \mathrm{~m}$ de altura, durante o período de 1998 à 2002 
Os meses de abril, maio e junho apresentam-se com os valores das velocidades médias diurnas menores que os demais, sendo o mês de abril o de ventos mais fracos. Com relação aos ventos noturnos, o mês de março foi o que apresentou menores valores médios de velocidade. De agosto a novembro os ventos sopram com intensidade superior à média, sendo que o mês de setembro é o de ventos com velocidades mais elevadas, tanto noturnas quanto diurnas, o que também foi verificado por MARQUES JUNIOR et al. (1995) e MARTINS (1993) para a cidade de Pelotas. Já de janeiro a julho os ventos são menos intensos, comportamento semelhante ao observado para a região de Ribeirão Preto por TUBELIS e NASCIMENTO (1984) e por TARIFA e ARMANI (2000) para a cidade de São Paulo.

\section{CONCLUSÃO}

Na maior parte do ano há predominância de ventos vindos de sudeste (SE), com exceção do período de dezembro - fevereiro, quando também há predominância de ventos noroeste $(\mathrm{NW})$, período em que a velocidade média diária começa a diminuir.

De agosto a novembro os ventos sopram com intensidade superior a média, sendo que o mês de setembro é o de ventos com velocidades mais elevadas, tanto noturnas quanto diurnas. De janeiro a julho os ventos são menos intensos, sendo o mês de abril o de menores velocidades médias.

O comportamento da velocidade dos ventos foi muito variável no período, havendo um aumento médio de $40 \%$ nas velocidades dos ventos diurnos em relação aos noturnos.

\section{REFERÊNCIAS BIBLIOGRÁFICAS}

DAS, D.K. et al. Environmental Baseline Data Collection at Diablillos Property, Salta, Argentina. In: Argentina Mining 98. Mendoza, Argentina, 1998, 15p.

GALVAni, E.; KLOSOWSKI, E. S. CUNHA, A.R. Caracterização da direção predominante do vento em Maringá, PR. Revista Brasileira de Agrometeorologia. Santa Maria, v. 7, n. 1, p. 81-90, 1999.

MACHADO, F.P. Contribuição ao estudo do Clima do Rio Grande do Sul. Rio de Janeiro, Conselho Nacional de Geografia, 1950. $91 \mathrm{p}$.

MARQUES JUNIOR, S., VALADÃO, L.T., VIEIRA, A.R.R., et al. Análise dos dados de vento para a região de Botucatu - SP utilizando a distribuição Beta. Revista Brasileira de Agrometeorologia, Santa Maria, v. 3, p. 129-132, 1995.
MARTINS, D. O comportamento dos ventos na região de Botucatu, SP. In: CONGRESSO BRASILEIRO DE ENGENHARIA AGRÍCOLA, 22, 1993, Ilhéus. Anais... Ilhéus: Sociedade Brasileira de Engenharia Agrícola / CEPLAC, 1993. $1413 \mathrm{p}$.

MORENO, J. A. Clima do Rio Grande do Sul. Porto Alegre Secretaria da Agricultura do Estado do Rio Grande do Sul, Diretoria de Terras e Colonização, Secção de Geografia, 1961.

NIMER, E. Climatologia do Brasil. Instituto Brasileiro de Geografia e Estatística, Rio de Janeiro, 1989. 421p.

PARKER, M.J.; WEBER, A.H.; BUCKLEY, R.L. Short Term Climatological Wind Data as a Tool for Wind Forecasting. Weather and Forecasting. American Meteorological Society, Boston, 2004.

PEREIRA, A.R.; ANGELOCCI, L.R.; SENTELHAS, P.C. Agrometeorologia: fundamentos e aplicações práticas. Guaíba.: Livraria e Editora Agropecuária, 2002. 478 p

REIS, B. G.; BERLATO, M. A. Aspectos gerais do clima do Estado. Porto Alegre, Instituto Nacional de Colonização e Reforma Agrária, 1972, v. 1, 187 p.

SENAMBI - Serviço Nacional de Meteorologia e Hidrologia del Peru. Aprendendo Meteorologia. In: http://www.senambi. gob.pe.pefaprediendo/apen_meteo.htm. 2004.

SILVA,J.B.; ZANUSSO,J.F.; SILVEIRA,D.L.M. Estudo da velocidade e direção dos ventos em Pelotas, RS. Revista Brasileira de Agrometeorologia, Santa Maria, v.5, n.2, p227-235, 1997.

TARIFA, J. R. ; ARMANI, G. . As Unidades Climáticas Urbanas da Cidade de São Paulo. In: José Roberto Tarifa. (Org.). Atlas Ambiental do Município de São Paulo. São Paulo, 2000, v. CD-Rom

TUBELIS, A.; NASCIMENTO, F. C. L. do. Meteorologia descritiva. Fundamentos e aplicações brasileiras. São Paulo, Nobel, 1984. 374p.

VENDRAMINI, E.Z. Distribuições probabilísticas de velocidades do vento para avaliação do potencial energético eólico. Botucatu: UNESP, 1986. 110 p. Tese (Doutorado em Agronomia). Curso de Pós-Graduação em Agronomia, Faculdade de Ciências Agronômicas, Universidade Estadual Paulista, 1986.

WAGNER, C.S. Velocidade e direção predominante dos ventos no Estado do Paraná. Londrina: Instituto Agronômico do Paraná, 1989. 56 p. (Boletim técnico, 26).

CARRER, T.; GARCIA,A.. Classificação Climática ParaACidade De Ituverava/SP. Nucleus, América do Sul, V.4,p 11 10, 2007. 